\title{
Global Remote Operation of Intelligent Space Robot Assistants
}

\author{
Daniel Leidner, ${ }^{1}$ Peter Schmaus, ${ }^{1}$ Florian Schmidt, ${ }^{1}$ Benedikt Pleintinger, ${ }^{1}$ \\ Ralph Bayer, ${ }^{1}$ Adrian S. Bauer, ${ }^{1}$ Thomas Krueger, ${ }^{2}$ Neal Y. Lii ${ }^{1}$
}

\author{
${ }^{1}$ Institute of Robotics and Mechatronics, German Aerospace Center (DLR), Wessling, Germany, \{first.last $\} @$ dlr.de \\ ${ }^{2}$ Human Robot Interaction Lab, European Space Agency (ESA), Noordwijk, The Netherlands, \{first.last\}@esa.int
}

\begin{abstract}
Intelligent robotic coworkers are considered a valuable addition in many application areas. This applies not only to terrestrial domains, but also to the exploration of our solar system. As humankind moves toward an ever increasing presence in space, infrastructure has to be constructed and maintained on distant planets such as Mars. AI-enabled robots will play a major role in this scenario. The space agencies envisage robotic co-workers to be deployed to set-up habitats, energy, and return vessels for future human scientists. By leveraging AI planning methods, this vision has already become one step closer to reality. In the METERON SUPVIS Justin experiment, the intelligent robotic coworker Rollin' Justin was controlled from Astronauts aboard the International Space Station (ISS) in order to maintain a Martian mock-up solar panel farm located on Earth to demonstrate the technology readiness of the developed methods. For this work, the system is demonstrated at AAAI 2019, controlling Rollin' Justin located in Munich, Germany from Honolulu, Hawaii.
\end{abstract}

In the near future, the major space agencies plan to revisit Moon and start the manned exploration of Mars. These ambitious goals are facing hazardous environments on the surface of the celestial bodies, which makes it difficult for humans to install a permanent base on-site. A possible solution to provide habitats, energy, breathable air and a return vehicle beforehand is given with the deployment of intelligent service robots that are controlled from a spacecraft in orbit. A prototype for such an agent is the humanoid robot Rollin' Justin (Borst et al. 2009). Dexterous manipulation capabilities paired with advanced reasoning mechanisms make it capable of setting up the required infrastructure in order to pave the way for human scientists. At its core, AI based planning methods enable it to serve as a coworker for astronauts, rather than a tool. While the robot performs the commanded tasks autonomously, the astronauts orbiting the target planet or moon can focus on scientific work. Intervening in robotic operation is only necessary to provide new objectives. This paper presents the methods developed during the METERON SUPVIS Justin space robotics experiments, which was conducted by astronauts on board the International Space Station (ISS), commanding a robot coworker on Earth as an analog celestial site.

Copyright (C) 2019, Association for the Advancement of Artificial Intelligence (www.aaai.org). All rights reserved.

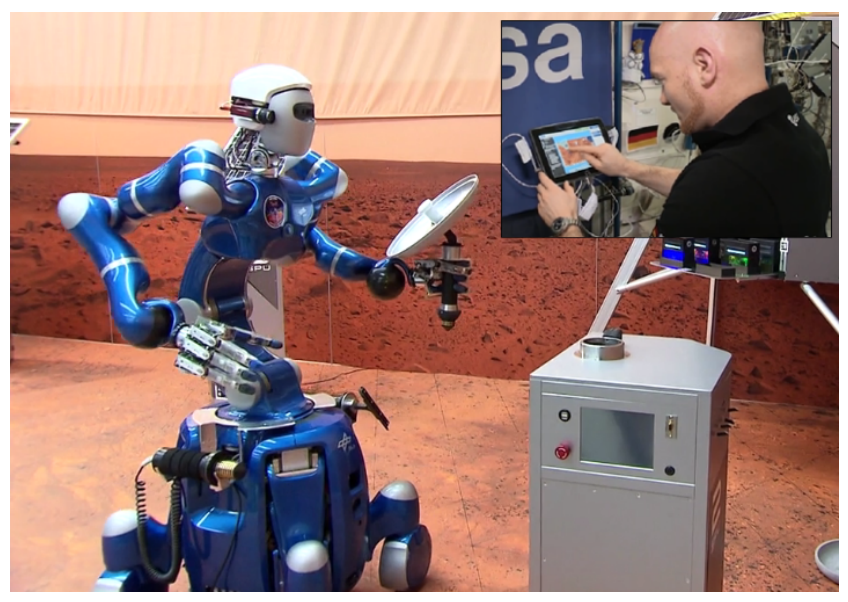

Figure 1: Rollin' Justin autonomously installing an antenna as response to the astronauts command during the final METERON SUPVIS Justin crew session on August 17, 2018.

\section{METERON SUPVIS Justin}

The SUPVIS Justin experiments explore teleoperation of extraterrestrial service robots with supervised autonomy (Lii et al. 2017) within the Multi-Purpose End-To-End Robotic Operation Network (METERON) project. Here, the robot serves as a coworker on the planetary surface for the astronaut in orbit (see Figure 1), who communicates via abstract commands and replies, rather than through detailed robot trajectories. The robot's local intelligence enables it to perform partially autonomous reasoning and execution. This relieves the astronaut of significant mental and physical workload.

Three crew sessions were carried out between 2017 and 2018. Ten experiment protocols have been conducted by a total of five NASA and ESA astronauts, of whom only two were fully trained prior to mission. Two astronauts were introduced to the systems by their crew mate interactively during the experiment. One astronaut, with no experience using the telerobotic system, was able to effectively command the robot thanks to the intuitive UI, and limited guidance from ground in a starting protocol. He was subsequently able to solve the most complex task in the mission. This proves that the proposed approach is suitable to create an intuitive user experience by exploiting the local intelligence of the robot. 


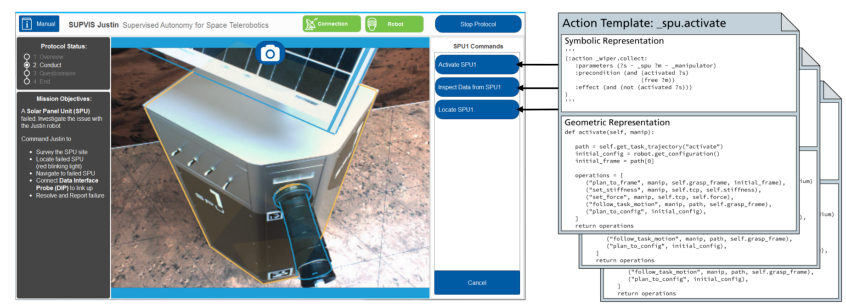

Figure 2: Commands in the tablet application (left) are authorized based on available Action Templates (right) and the current task context.

\section{Astronaut-Robot Collaboration Concept}

In the reasoning framework of Rollin' Justin, all actions are implemented by means of so called Action Templates (Leidner 2017), which constitute an object-centric representation of robot capabilities, combining symbolic and geometric features. That is, each object in the world state of the robot affords a set of available actions to the robot, providing the main building blocks for the reasoning apparatus of the robot. The symbolic representation of Action Templates is provided in Planning Domain Definition Language (PDDL) (Ghallab et al. 1998) which allows us to use state-of-the-art symbolic planning tools such as (Helmert 2006).

Action Templates are automatically parsed to create a list of possible robot commands made available in the astronauts tablet application on orbit (see Figure 2). Based on this, the astronaut has to decide on how to instrument the robot to achieve the mission objective. To do so, the astronaut is in charge of the robots capabilities to survey the environment (i. e. the possibility to alter the camera view), as well as to relocate the robot in the remote environment (i. e. navigate toward a target destination). Once the robot is close enough to reach a particular object, the astronaut can initiate the interaction by selecting the highlight object in the camera view.

While longer communication times from Mars to Earth may prevent ground control from directly intervening with the robot or the astronaut in real-time, it is possible to guide the astronaut by restricting the available robot actions to a necessary minimum. For example, it may be advised to limit the complexity of possible robot action chains to a certain level. That is, the robot is aware of the action sequence length $\Theta$ of all possible goal states currently reachable w. r. t. to the current state of the environment. Ground control may define $\Theta=1$ in order to have the actions of the robot frequently assessed by the astronaut.

\section{Technology Demonstration}

During the technology demonstration at AAAI 2019, the humanoid robot Rollin' Justin is controlled via the tablet application developed for the SUPVIS Justin experiments. The robot is located near Munich, Germany. The tablet computer is located in Honolulu, Hawaii. The distance between the locations is approximately $12215 \mathrm{~km}(7590 \mathrm{mi})$ resulting in communication times up to $250 \mathrm{~ms}$. In comparison, the ISS travels at an average altitude of $400 \mathrm{~km}(250 \mathrm{mi})$ which results in communication times below $25 \mathrm{~ms}$ using direct view connection (lasting for about 8 minutes) (Artigas et al. 2016). To guarantee extended communication times, the Tracking and Data Relay Satellite (TDRS) network was utilized during SUPVIS Justin, where communication times above $800 \mathrm{~ms}$ are nominal. Accordingly, the AAAI location serves as an intermediate benchmark for the remote operation of space assistant robots.

The robot is commanded to maintain the solar panel farm constructed for the SUPVIS Justin experiments, which requires the robot to inspect the environment (e.g. identifying malfunctioned units), maintain the solar panels (e.g. cleaning them with a wiper), setting up new modules (e. g. installing an antenna), or even repairing broken components (e. g. exchanging computation units). With this, the technology demonstration showcases the most complex tasks conducted in collaboration with a remotely operated robot to date (Schmaus et al. 2018).

\section{Acknowledgments}

This work was conducted within the METERON project. We would like to thank our friends and colleagues at ESTEC, European Astronaut Centre, Columbus Control Centre, German Space Operations Center, and Danish Aerospace, without whom METERON SUPVIS Justin would not have been possible.

\section{References}

Artigas, J.; Balachandran, R.; Riecke, C.; Stelzer, M.; Weber, B.; Ryu, J.-H.; and Albu-Schaeffer, A. 2016. KONTUR-2: Force-Feedback Teleoperation from the International Space Station. In IEEE International Conference on Robotics and Automation (ICRA), 1166-1173.

Borst, C.; Wimböck, T.; Schmidt, F.; Fuchs, M.; Brunner, B.; Zacharias, F.; Giordano, P. R.; Konietschke, R.; Sepp, W.; Fuchs, S.; et al. 2009. Rollin'Justin - Mobile Platform with Variable Base. In Proc. of the IEEE International Conference on Robotics and Automation (ICRA), 1597-1598.

Ghallab, M.; Howe, A.; Christianson, D.; McDermott, D.; Ram, A.; Veloso, M.; Weld, D.; and Wilkins, D. 1998. PDDL - The Planning Domain Definition Language. AIPS98 Planning Committee 78(4):1-27.

Helmert, M. 2006. The Fast Downward Planning System. Journal of Artifcial Intelligence Research 26:191-246.

Leidner, D. 2017. Cognitive Reasoning for Compliant Robot Manipulation. Ph.D. Dissertation, University of Bremen.

Lii, N. Y.; Leidner, D.; Birkenkampf, P.; Pleintinger, B.; Bayer, R.; and Krueger, T. 2017. Toward Scalable Intuitive Teleoperation of Robots for Space Deployment with the METERON SUPVIS Justin Experiment. In Proc. of the 14th Symposium on Advanced Space Technologies for Robotics and Automation (ASTRA).

Schmaus, P.; Leidner, D.; Krueger, T.; Schiele, A.; Pleintinger, B.; Bayer, R.; and Lii, N. Y. 2018. Preliminary Results of the METERON SUPVIS Justin Space-Robotics Experiment. In In Proc. of the IEEE/RSJ International Conference on Intelligent Robots and Systems (IROS). 